\title{
No need to reduce water
}

Nat. Mater. https://doi.org/10.1038/s41563-020-

0667-y (2020)

Owing to the non-flammability of aqueous electrolytes, aqueous batteries have safety advantages over non-aqueous counterparts. However, the stable operating voltage window of water is merely $1.23 \mathrm{~V}$, beyond which water electrolysis takes place, leading to severe deterioration of electrode structures and battery performance. This intrinsic property of water significantly limits the energy density of aqueous batteries. Decreasing the water content by using highly salt-concentrated (typically larger than $20 \mathrm{~mol} \mathrm{~kg}^{-1}$ ) electrolytes is the most effective approach to expand the voltage window and thus to enhance the energy density, but high viscosity and high cost are the major drawbacks of such electrolytes. Now, Yi-Chun Lu and colleagues from the Chinese University of Hong Kong report that adding poly(ethylene glycol) (PEG) in conventional aqueous electrolytes substantially expands the stable operating voltage to $3.2 \mathrm{~V}$, without having to increase the salt concentration.

The researchers explain that in the electrolyte, water is confined by its hydrogen bonding with PEG. Furthermore, due to the electron-donating effect of the alkyl groups in PEG, the ethereal oxygen atom in PEG has a higher electronegativity than the oxygen atom in water. This weakens the hydrogen bonding between PEG and water, but strengthens the $\mathrm{H}-\mathrm{O}$ covalent bond of water. The $\mathrm{H}-\mathrm{O}$ bond strengthening is key for the much higher electrochemical stability of water than in the conventional aqueous electrolytes. The researchers demonstrate that in an electrolyte containing a low concentration $\left(2 \mathrm{~mol} \mathrm{~kg}{ }^{-1}\right)$ of lithium bis(trifluoromethane)sulfonimide salt, the addition of PEG almost completely suppresses water electrolysis and leads to a high-voltage $\mathrm{Li}_{4} \mathrm{Ti}_{5} \mathrm{O}_{12} / \mathrm{LiMn}_{2} \mathrm{O}_{4}$ full cell.

Changjun Zhang

Published online: 20 May 2020

https://doi.org/10.1038/s41560-020-0626-5 